\section{Dependence of Electrotransport on Composition in Simple Liquid Binary Alloys}

\author{
S. J. Larsson, A. Lodding, T. Persson, \\ and C. ROXBERGH
}

Physics Department, Chalmers University of Technology

(Z. Naturforsch. 28, 314-315 [1973]; received 2 December 1972)

To Professor A. Klemm on bis sixtieth birthday

The phenomenon of electrotransport in a binary liquid alloy with perfectly miscible components is representable in terms of measurable resistivity and diffusivity parameters. The formulae are compared with experimental data from measurements of electrotransport in liquid $\mathrm{Na}-\mathrm{K}$. The predicted magnitudes as well as signs of the relative mobilities are satisfactorily borne out by the experiments.

The interest at this Institute in liquid metal electrotransport dates back to the early 1950-s, and is a consequence of Professor A. Klemm's stay at Gothenburg as Visiting Professor. His introduction of the concept of electron wind to explain the "Haeffnereffect" has greatly stimulated the research in the field, and contributed to start the systematic investigation of which the present paper is a part.

In a recent study of electrotransport in liquid $\mathrm{Na}-\mathrm{K}$ alloys ${ }^{1}$ an attempt was made to express, for any li- quid metal binary with perfectly miscible components and in terms of a few wellknown or measurable parameters, the temperature and composition dependence of the effective charge number $Z^{*}{ }_{A-B}$. This entity is defined by

$$
v_{\mathrm{A}-\mathrm{B}}=\left(D_{\mathrm{i}} / k T\right) \cdot Z{ }_{\mathrm{A}-\mathrm{B}} E e,
$$

where $v_{A-B}$ is the relative migration velocity of the two alloying components $\mathrm{A}$ and $\mathrm{B}$ in the field $E$, and $D_{\mathrm{i}}$ their inter-diffusion coefficient.

The relation arrived at $\left[\mathrm{cf} .{ }^{1} \mathrm{Eq} .(16)\right]$ can be written as

$$
\begin{array}{r}
-\bar{\varrho} Z_{\mathrm{A}-\mathrm{B}}^{*}=\left[\left(1-2 c_{\mathrm{A}}\right) \varrho^{* *_{0}}+\frac{1}{2} \Delta \varrho^{*{ }_{\mathrm{D}}}\right] \\
+\frac{D^{*_{\mathrm{A}}-D{ }_{\mathrm{B}}}}{D^{*{ }_{\mathrm{A}}}}\left[c_{\mathrm{A}}^{2} \varrho^{*}{ }_{0}+\frac{1}{2} \varrho \mathrm{D}_{\mathrm{B}}-\bar{\varrho}\right] .
\end{array}
$$

In the experiments on the $\mathrm{Na}-\mathrm{K}$ system, the last term is only between $2 \%$ and $15 \%$ of the whole. Here $D * A, B$ are the respective tracer diffusion coefficients for $c_{\mathrm{A}, \mathrm{B}}=0, c_{\mathrm{A}, \mathrm{B}}$ denoting the respective mole fractions. $\bar{Q}$ is the alloy resistivity; $\varrho^{*}$ is the resistivity increment on mixing $\mathrm{B}$ as impurity in pure $\mathrm{A}$, or vice versa (see Ref. ${ }^{2}$ ); $\frac{1}{2} 0 *$ D denotes the "defect resistivity" of mobile species, obtainable from studies of isotope electrotransport ${ }^{3}$; and $\frac{1}{2} \Delta \varrho^{*} \mathrm{D}$ is the difference in this entity between $\mathrm{A}$ and $\mathrm{B}$.
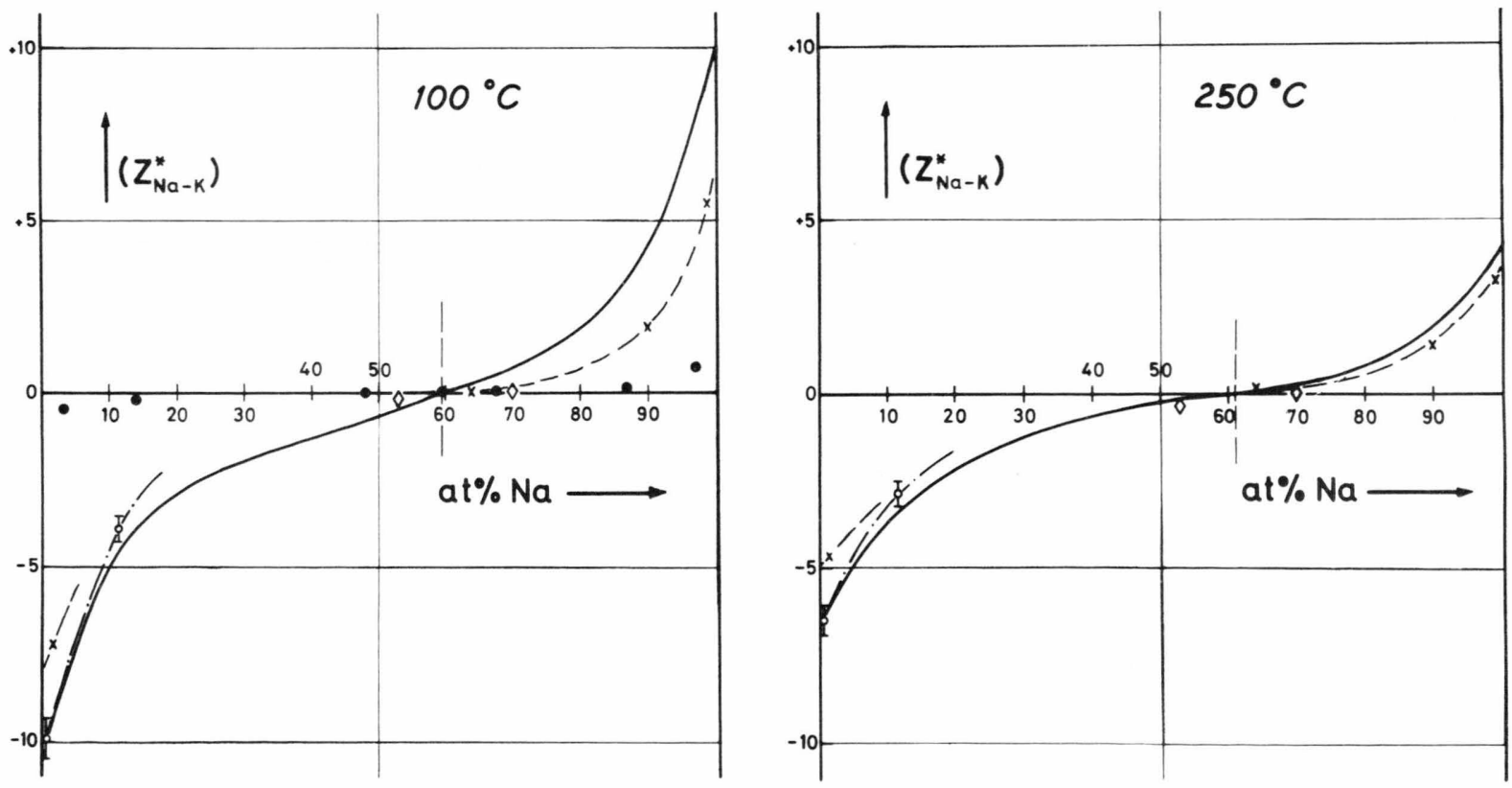

Fig. 1. The concentration dependence of relative effective charge numbers of electrotransport in liquid Na-K alloys at $100^{\circ}$ and $250^{\circ}$ C. Full curves: from Eq. 2 with $\varrho^{*}=1.35, \Delta \varrho^{*} \mathrm{D}=0.25$, $\varrho^{*} \mathrm{D} \mathrm{K}=0.6$. Broken curves: interpolated experimental. Empty circles: Ref. ${ }^{1}$; Full circles: Ref. ${ }^{6}$; Diamonds: Ref. ${ }^{7}$; Crosses: Reference ${ }^{8}$. 
In Ref. ${ }^{1}$ it was shown that the temperature dependence of $Z{ }_{A-B}$, as measured for liquid $\mathrm{Na}-\mathrm{K}$ at low $\mathrm{Na}$ concentrations, agreed very well with the above formulae, when the parameters $\varrho^{*} \simeq 1.35$ and $\frac{1}{2} \Delta \varrho^{*}$ D $\simeq 0.25$ (in $\mu \Omega \mathrm{cm} / 0 \%$ def.) were substituted. This $\varrho^{*}$. value is in good agreement with resistivity measurements $2,4,5$. The difference in $\frac{1}{2} o_{n}$ is at least qualitatively plausible: from Haeffner-effect measurements ${ }^{3}$ one has inferred $\frac{1}{2} \Delta Q^{*} \mathrm{n}=0.6 \pm 0.4 \mu \Omega \mathrm{cm} / \%$ for potassium, while the (so far unmeasured) corresponding term for sodium may be assessed to lie between 0 and 1.2 .

The purpose of this communication is to show that also the concentration dependence of $Z{ }^{*} \mathrm{~A}-\mathrm{B}$, as observed in several investigations of $\mathrm{Na}-\mathrm{K}^{1,6-8}$, is reasonably represented by the above equations, adopting the above given values of the parameters $\varrho^{*}{ }_{0}$ and $\varrho^{*} \mathrm{D}$.

The plots based on Eq. (2) are shown for two temperatures in Figure 1. For their construction the resistivity data were taken from Refs., ${ }^{4}$ and the diffusivity data from Reference ${ }^{9}$.

The figure also shows all hitherto obtained experimental data for $Z^{*} \mathrm{Na}-\mathrm{K}$ at $100^{\circ}$ and $250^{\circ} \mathrm{C}$. According to Ref. ${ }^{1}$, the points represented by filled circles ${ }^{6}$

1 C. Roxbergh, T. Persson, and A. Lodding, Phys. Chem. Liquids 4, 1 [1973].

2 N. F. Mott and H. Jones, The Theory of the Properties of Metals and Alloys, Oxford Univ. Press, New York 1958, Section 13.1.

3 A. Lodding, J. Phys. Chem. Solids 28, 557 [1967].

${ }^{4}$ Liquid Metals Handbook, J. Lyon ed., Navexos P 733 (Rev.), 1954.

5 J. F. Freedman and W. D. Robertson, J. Chem. Phys. 34, 769 [1961]. probably lie much too low, due to experimental shortcomings; the crosses ${ }^{8}$ are thought to be of the right order of magnitude but might be on the low side due to convection effects.

It is seen that the theoretical curves agree excellently with the low $\mathrm{Na}$ results, measured in Ref. ${ }^{1}$, and qualitatively also with those obtained at various compositions in References ${ }^{7,8}$. As regards the cross-over from negative to positive $Z{ }^{*} \mathrm{Na}-\mathrm{K}$, our equations predict it to lie at about $60 \% \mathrm{Na}$ and somewhat temperature dependent, which is in very good agreement with experiments ${ }^{6-8}$. This sign reversal of electrotransport in liquid binary alloys has been treated by phase-shift approach theory $8,10,11$ which, however, expects a cross-over in $\mathrm{Na}-\mathrm{K}$ at about $32 \% \mathrm{Na}$, rather than the experimentally observed $60 \%$. The formalism of Ref. ${ }^{1}$ is seen to lead to the essentially correct prediction, but only as a consequence of $\Delta \varrho^{*}$ D being positive, which is theoretically not a priori obvious and has not yet been proved experimentally; new Haeffner-effect measurements on the $\mathrm{Na}-\mathrm{K}$ system are now in preparation.

This work has been supported by the Swedish $\mathrm{Na}$ tural Science Research Council and the W. \& M. Lundgren Science Fund.
6 S. I. Drakin and A. K. Maltsev, Zh. fiz. Khim. 31, 2036 [1957].

7 J. C. Jousset and H. B. Huntington, Phys. Stat. Sol. 31, 775 [1969].

8 S. G. Epstein and J. M. Dickey, Phys. Rev. B 1 , 2442 [1970].

9 T. PERSSON and S. J. Larsson, Z. Naturforsch. in press.

10 D. L. Olsson, J. L. Blough, and D. A. Rigney, Scripta Met. 4, 1023 [1970].

11 D. L. Olsson, J. L. Blough, and D. A. Rigney, Acta Met. 20, 305 [1972]. 